\title{
The dilemma of using psychiatric medication in pregnancy. A case report and a review of literature
}

\author{
Mădălina Elena Prisacaru, Tudor Andrei Buțureanu, Cozmin Mihai
}

Mădălina Elena Prisacaru - M.D., Resident Psychiatrist, Institute of Psychiatry „Socola, Iași, România

Tudor Andrei Buțureanu - M.D., Gynecologist and Obstetrician, Department of Mother and Child, University of Medicine and Pharmacy, Iași, România

Cozmin Mihai - M.D., PhD, Psychiatrist, Institute of Psychiatry „Socola,, Iași, România

\begin{abstract}
The management of mental disorders in pregnancy represents, still, quite a controversial area in medicine. In case of women, the highest incidence of onset of a mental disorder overlaps the fertile period. Scientific literature indicates that over $50 \%$ of the women suffering from a mental illness become others at least once in their lives.

Putting in balance the risks of discontinuing of the psychiatric treatment in patients with severe mental disorders and the potential teratogenic risks to which the foetus is subjected, an increasing number of studies recommends the preservation of medication throughout the pregnancy. The risks entailed by an unmonitored pregnancy are the following: pre-eclampsia, premature birth, delayed intrauterine growth, low birth weight, death in utero or post-birth. Notwithstanding, many future mothers discontinue the psychiatric medication when finding out of the pregnancy, for fear of the teratogenic risk. Recent studies have reported that most antipsychotics do not have a marked influence on the evolution of pregnancy. Environmental factors such as smoking, obesity, alcohol, poor diet have a significant negative influence, in their turn. The pregnancy of a woman diagnosed with schizophrenia is high risk, reason for which close monitoring is imperious. In this analysis, I will synthesise the benefits and the risks of the psychiatric treatment administered in pregnancy and I will outline a case within our clinic, of a young woman with schizophrenia who followed the psychiatric treatment throughout her entire pregnancy.
\end{abstract}




\section{KEY WORDS:}

Pregnancy, antipsychotics, benzodiazepines, mood stabilisers, schizophrenia, congenital malformations, teratogenicity, mental disorders.

\section{THEORETICAL BACKGROUND}

The incidence of schizophrenia in the general population is $1-2 \%$, affecting men and women to the same extent, regardless of their culture of socioeconomic class. The age at onset for schizophrenia among women is $25-35$, which is alarming for the quality of life of the patients, because it overlaps the fertile period. Nonetheless, some studies report a lower rate of fertility among women with schizophrenia compared to women within the general population. Unfortunately, most of the times, pregnancy is not planned and desired; the future mothers are not aware of the risks to which they subject both themselves and the foetus. (1)

Antipsychotic medication involves the most complicated mechanisms of pharmacological action among the entire psychotropic medication. It influences the nervous system through various mechanisms. When they are administered during pregnancy, they cross the placental barrier and they reach the central nervous system of the foetus. Because they pass easily through the blood-brain barrier of the foetus, there is a risk of producing modifications both in utero and postnatally, on a long term of the nervous system of the foetus. The high level of antipsychotics detected in the amniotic fluid suggests the existence of potential teratogenic effects, namely a negative impact on the subsequent neurological development of the child. Penetrance at the level of the placental barrier varies by antipsychotic. Researchers find it hard to isolate definitely the effects of each antipsychotic because women suffering from psychiatric disorders have complex treatment regiments, (with mood stabilisers, benzodiazepines, serotonin reuptake inhibitors) and they are more likely to consume psychoactive substances. (2)

Conventional antipsychotics - also known as first-generation antipsychotics or typical antipsychotics - were used for the first time in 1950 in the treatment of psychosis. (3) The first efficient treatments for schizophrenia and other psychotic disorders emerged more than 60 years ago, from clinical observations, not from scientific research on the mechanisms of antipsychotic action. Therefore, the first antipsychotic drug was discovered by chance, when it was found that a substance with antihistaminic features (chlorpromazine) would have antipsychotic effects when administered to patients with schizophrenia. Once chlorpromazine turned out to be an efficient antipsychotic agent, it was tested experimentally to discover its mechanism of antipsychotic action. The "conventional" character of antipsychotics is provided by their property of blocking the D2 dopaminergic receptors, especially at the level of the mesolimbic dopaminergic pathway. This results in a mitigation of hyperactivity at the level of this pathway, considered the cause of positive symptoms in psychosis. All conventional antipsychotics have more or less the same efficacy in reducing the positive symptoms in psychosis among patients with schizophrenia, should the dosage be enough to block a significant number of D2 receptors at that level. For most conventional antipsychotics, the degree of binding the D2 receptors at the level of the mesolimbic pathway to obtain the antipsychotic effects is almost $80 \%$. (4) Unfortunately, to block a sufficient number of D2 receptors at the level of the mesolimbic dopaminergic pathway in order to supress the positive symptoms, the 
same number of D2 receptors must be blocked at the level of the entire brain, and this determines undesired adverse effects. For instance, $80 \%$ blocking of $\mathrm{D} 2$ receptors at the level of dorsal striatum determines extrapyramidal side effects, while at the level of the hypophysis gland, it determines hyperprolactinemia. Hence, there is a narrow therapeutic window between the threshold of antipsychotics efficacy and the one of side effects concerning the binding degree to the D2 receptors. Conventional antipsychotics include: chlorpromazine, cyamemazine, flupentixol, fluphenazine, haloperidol, loxapine, mesoridazine, perphenazine, pimozide, pipotiazine, sulpiride, thioridazine, thiothixene, trifluoperazine and zuclopenthixol. (5)

Atypical antipsychotics (second generation) are of a more recent date, but they also have fewer side effects. They are used in both the treatment of psychotic disorders and as adjuvant to treat anxiety and depression. Atypical antipsychotics have a clinical profile characterised by effects on the positive symptoms as strong as those of conventional antipsychotics, but with fewer extrapyramidal symptoms and lower hyperprolactinemia compared to the conventional ones. Hence, they are atypical in relation to the known properties of a classic, first-generation antipsychotic. Atypical antipsychotics include: aripiprazole, clozapine, olanzapine, quetiapine, ziprasidone, amisulpride, risperidone. (6) From a pharmacological perspective, atypical antipsychotics are defined as serotonin and dopamine antagonists, with an antagonist action on both the serotoninergic $5 \mathrm{HT}_{2 \mathrm{~A}}$ receptors and the $\mathrm{D}_{2}$ receptors. All $5 \mathrm{HT}_{2 \mathrm{~A}}$ receptors are postsynaptic and they are found in various cerebral regions. Those present at the level of cortical pyramidal neurons sunt excitatory and they may thus determine an increase in downstream glutamate release, i.e. the blocking or stimulation of these receptors regulates the level of dopamine downstream. In what concerns the release of prolactin, serotonin and dopamine have a mutually regulating role: one annuls the effect of the other. Hence, the stimulation of $5 \mathrm{HT}_{2 \mathrm{~A}}$ receptors determines the reversal of $\mathrm{D}_{2}$ receptors stimulation effects. The stimulation of $5 \mathrm{HT}_{2 \mathrm{~A}}$ receptors by serotonin, in any of the neural terminations win the substantia nigra, blocks dopamine release at the level of the striatum. But the antagonization of $5 \mathrm{HT}_{2 \mathrm{~A}}$ receptors by an atypical antipsychotic at the same levels stimulates the dopamine release downstream at the level of the striatum, thus reducing the extrapyramidal effects. (5)

The most commonly used antipsychotics in pregnancy are the following: olanzapine, risperidone and quetiapine, because no concrete cases of congenital malformations or problematic new born development were reported when administering these antipsychotics.

A first important step in studying the effects of antipsychotics on pregnant women and on newborns was the creation by Kulkarni in 2005 of the Australian National Register of Antipsychotic Medication in Pregnancy. The study included 147 women with diagnoses such as schizophrenia, acute psychotic disorder, bipolar mood disorder and schizoaffective disorder treated with antipsychotic medication and 100 new-borns. The future mothers were monitored once every six weeks throughout pregnancy, at birth, then at 6 weeks, at 12 weeks, at 6 months and one year postpartum. The initial working tools were anamnesis with personal physiological and pathological history (obstetrical history), family history, the history of the mental disorder, the psychiatric medication administered from the beginning 
Fring

to the present, the social inquiry, the Positive and Negative Syndrome Scale (PANSS). Subsequently, throughout the pregnancy, the following were monitored: the mother's weight, the results to the glucose tolerance tests and to the usual blood tests, thickness of the nuchal fold, and every complication emerges was written down. Every six weeks postpartum, PANSS was repeated and the Edinburgh Postnatal Depression Scale (EPDS). Furthermore, we have monitored closely the evolution of the newborns in the first year of life and we got better insight into the complications at birth, breastfeeding, respiratory distress syndrome and newborn withdrawal syndrome. The most frequently used antipsychotics were quetiapine, followed by olanzapine. The results were influenced by additional risk factors such as mother's smoking throughout the pregnancy $(35 \%$ compared to the average of $13.5 \%$ of the smoking expectant mothers in Australia), the diverse therapeutic regimen including antidepressants (43\% of the participants), mood stabilisers, consumption of illicit substances and alcohol.

The findings of the study are hard to interpret taking into account the risk factors and the complex medication associated, but out of the 147 pregnancies monitored, 142 newborns resulted. Out of the 142 participants in the study, 37\% had respiratory distress syndrome at birth, while $40 \%$ required special medical assistance. We highlight the idea that the onset of respiratory distress syndrome in newborns was 6 times higher if the mother took mood stabilisers. Data show that $18 \%$ of the children were born prematurely, but in what concerns birth weight, it was close to that of the general population. In addition, 8 congenital malformations out of 147 participants were recorded, namely a percentage of $6 \%$, compared to the rate of congenital malformations in Austria: $3.1 \%$.
The congenital malformations were caused by the administration of certain antipsychotics. In case of using Quetiapine, two children were born with malformations, i.e. a newborn with pulmonary atresia and atrial septal defect, and the other with cheiloschisis, palatoschisis and encephalocele. When associating Quetiapine and Zuclopenthixol, a case of atrial septal defect was reported. When administering Risperidone, a newborn was born with renal malformations and tarus varus and a case of CHARGE syndrome (CHARGE syndrome is a genetic syndrome caused by a mutation of the CHD7 gene on chromosome 8). In case of Olanzapine, a case of congenital hip dysplasia was reported. In case of Clozapine treatment, two cases were reported: a child with three malformations (craniosynostosis,

hypospadias, hypertelorism) and another one with gastroschisis and horseshoe kidneys. Hence, there is no unequivocal data to connect the congenital malformations emerged to the antipsychotic medication administered, but the study provided conclusive data proving the correlation between antipsychotics and the following adverse effects: gestational diabetes, significant weight gain throughout the pregnancy, respiratory distress syndrome of the newborn and withdrawal syndrome to antipsychotic medication. Kulkarni monitored the children in the first year of life and he found no psychomotor development delays, but after this age, many subjects abandoned the study, so there is no long-term data. (7)

Monitoring a woman with severe mental illness throughout the pregnancy and postpartum is a challenge for the physicians, and the information about the potential longterm effects of antipsychotics on the foetus are limited.

A prospective study on 1309 pregnant women on phenothiazine, compared to 50.282 non- 
exposed pregnant women, found no evidence that it would increase the risk of congenital malformations, intrauterine or neonatal death.(8) Another study carried out in Massachusetts that assessed the safety of second-generation antipsychotics among pregnant women has shown that 3 out of 214 children born from mothers who took secondgeneration antipsychotics had congenital malformations compared to one child out of 89 within the control group. The medication comprises aripiprazole, asenapine, clozapine, iloperidone, lurasidone, olanzapine, paliperidone, quetiapine, risperidone and ziprasidone.

From a cohort of 1,360,101 pregnant women benefitting from Medicaid in the period 20002010 , a total of $9,258(0.69 \%)$ took atypical antipsychotics, i.e. $733(0.05 \%)$ received the second generation antipsychotic medication throughout their first trimester of pregnancy. The future mothers suffered from schizophrenia, acute psychotic disorder, depression, anxiety, ADHD, bipolar disorder. The exposed women were not more likely to record foetal congenital malformations, except for those exposed to risperidone. As for cardiac malformations, the results were similar. After assessing each antipsychotic, a slight risk increase was found for congenital malformations and cardiac malformations in case of risperidone treatment. A research study conducted in 1995-2010, on 416 pregnant women in the UK who received antipsychotic medication throughout their entire pregnancy has found no increased risk of giving birth to a child with serious congenital malformations in case of women on antipsychotics, compared to the control group of 318 women, as well as 670 women on antipsychotics before getting pregnant, but who discontinued it throughout the pregnancy. Out of the 290 women who took antipsychotic medication in pregnancy, 10
(3.4\%) gave birth to children with congenital malformations, compared to 11 women who were not compliant with antipsychotic treatment out of $492(2.2 \%)$, as well as 4,162 out of 210,966 (2\%), accounting for the control group. Therefore, the difference was insignificant, considering also the possible influence of associated medication, of the general health state and of the environmental factors. (9)

Opinions are divergent concerning the neonatal adverse effects of antipsychotics. Some researchers argue that they noticed neonatal adaptation difficulties among children exposed in utero to antipsychotics, such as: low Apgar score, hypoglycaemia, agitation, muscle hypotonia, drowsiness, respiratory failure (7) extrapyramidal symptoms (10). For instance, after the analysis the Australian National Register of Antipsychotic Medication in Pregnancy, out of the 142 children exposed in utero to antipsychotics, $37 \%$ suffered from respiratory distress at birth. (2) On the other hand, there are researchers who believe that schizophrenia per se features an array of adverse effects independent from the use of antipsychotics, such as: placental abnormalities, high risk of miscarriage, prematurity, preeclampsia, low birth weight, intrauterine growth delay, respiratory distress syndrome, neonatal hypoglycaemia, low Apgar score, spontaneous miscarriage. It is impossible to determine whether such adverse effects are caused by the mental disorder, by the antipsychotic medication or by the mother's unhealthy behaviour, because many of them drink, smoke, do drugs and have a dysfunctional relation with health services. (11) On the other hand, in case of numerous pregnant patients, the mental state requires a more complex therapeutic regimen. Th most common drugs are benzodiazepines, to treat anxiety. Researchers argue that 
p.s.

benzodiazepines account for $85 \%$ of the psychotropic medication prescribed in pregnancy. Nonetheless, they should be avoided during the first and the third trimester of pregnancy because some studies have found evidence of teratogenic features. The studies conducted on subjects taking Diazepam mentioned adverse effects on the newborns, such as limb abnormalities, stenosis, anal and rectal atresia, cardiovascular malformations, cheiloschisis and palatoschisis (12). Studies concerning Lorazepam and Bromazepam have found the presence of digestive malformations in the newborns exposed. The benzodiazepines with the least effects on the foetus proved to be Clonazepam and Alprazolam (13). In what concerns the use non-benzodiazepine hypnotic agents, there is no conclusive data to attest increased risk of congenital malformations in the children exposed. Nonetheless, studies carried out on a small number of mothers exposed to Zolpidem reported adverse effects, such as: newborns with LBW- low birth weight, SGA-small gestational age, caesarean birth. (14) Whereas, in what concerns antipsychotic medication, benzodiazepines and nonbenzodiazepine hypnotics, opinions concerning teratogenicity are divergent, reason for which they may be prescribed in pregnancy under certain circumstances, when we discuss anticonvulsants, most researchers prohibit their use among pregnant women. Anticonvulsants are definitely teratogenic: a 2009 study indicates a malformation rate of $2.7 \%$ for lamotrigine, $2.9 \%$ for carbamazepine and $8.7 \%$ for sodium valproate. (15) A study carried out in 2004 by Yonkers and his collaborators features the most common adverse effects among the newborns who were exposed in utero to sodium valproate: neural tube defects, cardiac malformations, facial malformations, limb malformations, urogenital malformations, tachycardia, withdrawal syndrome (irritability, muscle hypotonia, tremor, hepatotoxicity, hypoglycaemia). (16) Bromley and his collaborators studied the evolution of the first years of life exposed in utero to sodium valproate. The most notable adverse effects identified were low IQ, speech disorders, global neuromotor development delay and behavioural disorders. (17)

\section{GENERAL PRESENTATION OF THE CASE}

This case refers to a female patient aged 35 ani, from a rural area, unemployed, Romanian citizenship, with higher education, in a relationship, with a child from the previous marriage, in the records of the "Socola" Institute of Psychiatry since 2012, when she was diagnosed with Acute psychotic disorder postpartum. Throughout the course of the disorder, she was given antipsychotics (risperidone, olanzapine), benzodiazepines (lorazepam, bromazepam, clonazepam), mood stabilisers (valproic acid), but she went through numerous hallucinatory delusional decompensations due to non-compliance to the psychiatric treatment. After each discharge from the psychiatric clinic, the patient was prescribed birth control pills throughout the psychiatric treatment and she was well informed on the risks of carrying a pregnancy with the diagnosis of schizophrenia and given her complex pharmacological regimen. Nonetheless, the patient was admitted in psychiatric emergency for psychomotor agitation, hallucinatory delusional phenomena, as well as verbal and physical hetero-aggressiveness; she was also found to be 16 weeks pregnant. The arbitration Committee of the hospital decided that the gestational age was too far along for an abortion and they recommended the continuation of the treatment prescribed by her psychiatrist. The treatment comprised Olanzapine $10 \mathrm{mg}$ in two doses per day and 
Bromazepam $3 \mathrm{mg}$ in three doses per day, increasing progressively to $9 \mathrm{mg}$ per day, subsequently decreased and eventually discontinued within a month. She was admitted to the maternity and monored by a gynaecologist, who decided to perform a caesarean section at 38 weeks and 6 days. The conclusions of the echocardiographic exam have shown that the foetus had cranial presentation with fundic and anterior placenta. The amniotic fluid was in normal amount and the estimated weight of the foetus was 3,155 g. Cardiotocographic exams have shown normal foetal heartbeat, without arrhythmias. Both during her hospital stay and throughout her entire pregnancy, the patient was regularly monitored by the gynaecologist and the psychiatrist, but the patient did go undergo all tests (double test, foetal morphology, T1, T2, T3) recommended throughout pregnancy; the patient came to see the specialists when her pregnancy was already 16 weeks.

The patient gave birth via caesarean section under sub rachianaesthesia - due to the uterus scarring diagnosis - to a living female weighing 2,870 g; the Apgar score was 9, in the $50^{\text {th }}$ percentile, without clinical signs of congenital abnormalities. The evolution of the mother and of the newborn was favourable postoperatively. The gynaecologist in collaboration with the psychiatrist decide on ablactating with Bromocriptine due to the patient's risk of breastfeeding her newborn. One week after the birth, the patient returned to the psychiatrist for psychomotor agitation, reason for which valproic acid - $500 \mathrm{mg}$ per day - was added.

The values of lab works ranged within normal limits, except for slight anaemia, with a haemoglobin of $9.9 \mathrm{~g} / \mathrm{dl}$ postpartum, dropping from $11 \mathrm{~g} / \mathrm{dl}$. She was discharged 8 days postpartum with a recommendation to return to the maternity regularly for follow-up in a month. On this occasion, after counselling and getting consent from the patient, the gynaecologist decided to insert an intrauterine device as a birth-control method.

\section{CONCLUSIONS}

There are still not enough studies certifying the safety of using antipsychotic medication in pregnancy. Nonetheless, numerous studies have reported that the discontinuation of treatment throughout pregnancy jeopardizes the life of both mother and foetus, reason for which it is recommended to maintain antipsychotic treatment during pregnancy.

Most antipsychotics do not have a significant influence on the evolution of pregnancy; environmental factors such as smoking, obesity, alcohol, unhealthy diet have an important negative influence, as well.

The wish to perpetuate the species is one of the most powerful human instincts and, at the same time, a fundamental right of each woman, regardless of her mental state. It is important to focus on improving the quality of life for our female patients, to prescribe psychiatric medication pursuant to each case, to notify the patients concerning the potential risks and to encourage their insertion and integration in the society.

\section{ACKNOWLEDGEMENTS AND DISCLOSURES}

The authors state that they are no declared conflicts of interest regarding this paper. 


\section{REFERENCES}

1. Einarson, Adrienne, Rada Boskovic. Use and Safety of Antipsychotic Drugs During Pregnancy. Journal of Psychiatric Practice, vol. 15, no. 3, 2009, pp. 183-192., doi:10.1097/01.pra.0000351878.45260.94.

1. Ornoy, Asher, et al. Antidepressants, Antipsychotics, and Mood Stabilizers in Pregnancy: What Do We Know and How Should We Treat Pregnant Women with Depression. Wiley Online Library, John Wiley Ltd, 2017, onlinelibrary.wiley.com/doi/full/10.1002/bdr2.1079.

2. Stahl SM. Describing an atypical antipsychotic: receptor binding and its role in pathophysiology. Journal Clinical Psychiatry vol. 5, no.3, 2003, pp 5-10.

3. Chamberlin SR, Muller U, Blackwell AD, et al. Neurochemical modulation of response inhibition and probabilistic learning in humans. vol. 311, 2006, pp 861-863

4. Stahl, Stephen M., et al. Stahl's Essential Psychopharmacology: Prescriber's Guide. Cambridge University Press, 2017.

5. Aguilar, Mark L. Antipsychotic Drugs: Classification, Pharmacology and Long-Term Health Effects. Nova Science Publishers, 2016.

6. Kulkarni, Jayashri, et al. Antipsychotic Use in Pregnancy. Expert Opinion on Pharmacotherapy, vol. 16, no. 9, 2015, pp. 1335-1345., doi:10.1517/14656566.2015.1041501.

7. Slone, Dennis, et al. Antenatal Exposure to the Phenothiazines in Relation to Congenital Malformations, Perinatal Mortality Rate, Birth Weight, and Intelligence Quotient Score.American Journal of Obstetrics and Gynecology, vol. 128, no. 5, 1977, pp. 486-488., doi:10.1016/0002-9378(77)90029-1.

8. Petersen, Irene, et al. Risks Associated with Antipsychotic Treatment in Pregnancy: Comparative Cohort Studies Based on Electronic Health Records. Schizophrenia Research, vol. 176, no. 2-3, 2016, pp. 349-356.,

9. Howard, L. M. Atypical Antipsychotic Use during the First Trimester of Pregnancy May Not Increase Major Malformations. Evidence-Based Mental Health.2005;8(4):115-116.

10. Teodorescu A et al. Dilemma of Treating Schizophrenia during Pregnancy: a Case Series and a Review of Literature. BMC Psychiatry.2017;17(1)

11. Czeizel, Andrew E, et al. Short-Term Oral Diazepam Treatment during Pregnancy. Clinical Drug Investigation.2003;23(7):451-462.

12. Lin, Angela E., et al. Clonazepam Use in Pregnancy and the Risk of Malformations. Birth Defects Research Part A: Clinical and Molecular Teratology.2004;70(8):534-536.

13. Sharma, Sunil, Rose Franco. Sleep and Its Disorders in Pregnancy. Indian Journal of Sleep Medicine.2006;1(2):72-77.

14. Walker, Sp, et al. The Management of Epilepsy in Pregnancy. BJOG. 2009;116(6): 758-767.

15. Yonkers KA, Wisher KL, Stowe Z, Leibenluft E, Miller L, et al. Management of bipolar disorder during pregnancy and the postpartum period. American Journal Psychiatry. 2004; 161:608-20.

16. Bromley, R. L., et al. The Prevalence of Neurodevelopmental Disorders in Children Prenatally Exposed to Antiepileptic Drugs. Journal of Neurology, Neurosurgery, Psychiatry.2013; 84(6):637-643.

\section{Correspondence:}

Cozmin Mihai,

M.D., PhD, Psychiatrist, Institute of Psychiatry „,Socola,, Iași, România, dr.cozminmihai@gmail.com

Submission: 31 mar 2020

Acceptance: 29 may 2020 